\title{
Perfil energético-nitrogenado en caprinos del nordeste argentino según estaciones en dos años con diferente régimen pluvial
}

\author{
Brem, J.J.; Ortíz, M.L.; Trulls, H.E.; Zach, A.; Brem, J.C. \\ Laboratorio de Análisis Fisicoquímicos anexo a la Cátedra de Biofísica, Facultad de Ciencias Veterinarias de \\ la Universidad Nacional del Nordeste, Sargento Cabral 2139, Corrientes (3400), Argentina. Tel. (03783)-430101. \\ E-mail: jjbrem@vet.unne.edu.ar
}

\begin{abstract}
Resumen
Brem, J.J.; Ortíz, M.L.; Trulls, H.E.; Zach, A.; Brem, J.C.: Perfil energético-nitrogenado en caprinos del nordeste argentino según estaciones en dos años con diferente régimen pluvial. Rev. vet. 22: 2, 100-104, 2011. Las deficiencias nutricionales generalmente se manifiestan en forma subclínica con alteraciones de algún índice productivo y solo pueden ser detectadas con ayuda del laboratorio. La determinación e interpretación de ciertos parámetros bioquímicos permiten evaluar el estado metabólico del animal, transformándose en una valiosa herramienta para el diagnóstico poblacional del rodeo. En el nordeste argentino la producción caprina es de tipo familiar y extensiva; la alimentación se basa casi exclusivamente en el pastizal natural, arbustales y montes, con gran variabilidad estacional de la calidad y cantidad de la oferta forrajera, supeditada al comportamiento del régimen pluviométrico. Como parte de un proyecto mayor, se presenta aquí el comportamiento estacional de algunos indicadores bioquímicos en el ganado caprino de la región, determinados durante dos años consecutivos. El trabajo se llevó a cabo en establecimientos de las localidades de Riachuelo, Paso de la Patria y Empedrado en la Provincia de Corrientes. El muestreo se efectuó en forma estacional sobre un total de 150 animales de todas las categorías, durante dos años con registros pluviométricos muy diferentes: $2008(950 \mathrm{~mm})$ y $2009(1.490 \mathrm{~mm})$. Se determinaron parámetros sanguíneos del perfil energético-nitrogenado a través de técnicas espectrofotométricas de absorción molecular. Todos los parámetros del perfil lipídico se incrementaron durante el verano del año más lluvioso; colesterol HDL y total también lo hicieron en la primavera más seca y este último en el invierno más lluvioso. Colesterol LDL se incrementó en invierno del primer año y en otoño del segundo. En invierno y primavera del año más seco los animales cursaron con descensos de proteínas totales y albúminas, respectivamente. La hipoglucemia se manifestó en el otoño e invierno más seco y durante el verano de ambos años. Se concluye que la variación estacional registrada en algunos indicadores del perfil energético-proteico podría adjudicarse a cambios en la pastura ocasionados por las grandes diferencias pluviométricas observadas entre ambos años.
\end{abstract}

Palabras clave: cabra, perfil energético-nitrogenado, lluvias, nordeste argentino.

\begin{abstract}
Brem, J.J.; Ortíz, M.L.; Trulls, H.E.; Zach, A.; Brem, J.C.: Energy-nitrogen profile in goats from northeast Argentina according to seasons in two years with different rainfall patterns. Rev. vet. 22: 2, 100-104, 2011. Seasonal behaviour of some biochemical indicators from goats of North-eastern Argentina during two consecutive years, are presented. Nutritional deficiencies are commonly subclinic, they affect some productive indexes, and can only be detected through laboratory analysis. The determination and interpretation of certain biochemical parameters allow to asses the metabolic state of the animal, being this a valuable tool for the diagnosis of the rodeo. Goat production for this region is familiar and extensive; food is based almost exclusively on natural grass, shrubs and low vegetation. There is great seasonal variability that affects the quality and quantity of forage supply, and that depends of the performance of the rainfall regime. The work was carried out in the Province of Corrientes (Argentina). Sampling was carried out in each season on a total of 150 animals from different categories, for two years with very different rain records: $950 \mathrm{~mm}$ (2008) and 1,490 $\mathrm{mm}$ (2009). Blood parameters of energetic-nitrogen profile were determined by molecular absorption spectrophotometric techniques. All the parameters of the lipid profile were in-
\end{abstract}


creased during summer of the rainy year. Total and HDL cholesterol were higher during the driest spring, and HDL cholesterol during the rainy winter. LDL cholesterol was increased during the winter of the first year and autumn of the second one. During winter and spring of the driest year, animals had hypoproteinemia and hypoalbuminemia. Low values of glycemia were detected during the driest autumn and winter and in summer of both years. It can be concluded that seasonal variations in some indicators of the energetic-protein profile were caused by changes in grass composition attributable to the pluviometric differences in both years.

Key words: goat, energetic profile, nitrogen profile, rains, Northeast Argentina.

\section{INTRODUCCIÓN}

La crianza y explotación de cabras es una actividad que, a través de la producción de carne y leche, incrementa la disponibilidad de proteínas de alto valor biológico; además es una fuente de ingresos para muchas familias ofreciendo una serie de ventajas, como ser: baja inversión de capital, necesidad de poco espacio, alta capacidad reproductiva y facilidad de manejo ${ }^{12}$.

Respecto a la alimentación caprina, la literatura científica disponible no es tan extensa como ocurre con otras especies domésticas y han resultado relativamente difíciles los esfuerzos para extrapolar los requerimientos de nutrientes derivados de estudios realizados sobre vacunos y ovejas, ya que las cabras son mantenidas con dietas forrajeras de distintas características morfogenéticas y el consumo voluntario de alimentos frecuentemente limita la producción ${ }^{19}$.

Las cabras son mas hábiles en la cosecha de follaje siendo capaces de mantenerse sobre las patas traseras para alcanzar la copa de los árboles llegando a alturas que sobrepasan $2 \mathrm{~m}$ del suelo e incluso logrando trepar árboles para consumir el follaje en una proporción mayor a lo estimable. La cosecha en los arbustos y árboles se concentra sobre hojas más que ninguna otra parte de la planta, alimentándose de especies muy diferentes lo que provoca una baja contribución en la cantidad individual ${ }^{26}$.

El valor nutritivo del follaje de las especies de árboles de hojas caducas es más alto que el de las especies herbáceas, fundamentalmente durante período de sequía estival donde el contenido de la fibra en la dieta es alto ${ }^{24,26}$. Los animales consumen las especies preferidas aunque se encuentren en baja disponibilidad, lo cual repercute en la tasa de ingestión ${ }^{6,18}$, sin embargo las especies mas disponibles son las primeras a ser cosechadas por las cabras ${ }^{23}$. Las cabras en relación a las ovejas incluyen mayor cantidad de árboles y arbustos en sus dietas, comparadas con herbáceas y gramíneas que son consumidas principalmente durante otoño y principios de invierno, cuando se ve reducido el "ramoneo" ${ }^{25,26 .}$

Frente a comunidades de gran heterogeneidad de gramíneas, herbáceas, arbustos y árboles, la cabra emplea más tiempo en escoger la parte de los vegetales a cosechar, eligiendo dentro de un rango más amplio de especies preferidas en relación a los ovinos y vacunos, donde las características de la selección del alimento es diferente para cada comunidad vegetal ${ }^{26}$.

Es así que las cabras tienen una gran capacidad de adaptación dependiendo de la vegetación disponible, siendo consideradas como consumidoras adaptativas con una selectividad media a alta, relacionada en parte a las características anatómicas de su boca, que muestra una amplia movilidad de los labios y lengua prensil. Las características nutricionales propias del comportamiento alimentario del caprino han determinado la dificultad de proporcionar una dieta con los nutrientes necesarios y que sea apetecible por parte de los animales para mantener una buena condición fisiológica y lograr niveles aceptables de producción ${ }^{23}$.

Para el caso de la producción caprina en el centrooeste de Chaco y Formosa es importante destacar que se realiza en forma extensiva y que la alimentación proviene del pastizal natural (pajonales, arbustos y montes), donde existe una gran variación de la oferta forrajera, no sólo estacional en cuanto a cantidad y calidad, sino también local y aún dentro del mismo potrero. En los sistemas de producción extensiva, la variación y escasez de los recursos alimenticios para el ganado se debe, entre otros a: manejo inadecuado del pastizal (sobrepastoreo), escasez de aguadas o mala distribución, falta de apotreramiento y precipitaciones insuficientes, generando como consecuencia una baja productividad de la majada ${ }^{27}$. Bajo estas condiciones, el caprino es una de las especies mejor adaptadas para transformar los pastizales en productos aptos para el hombre: carne, leche, cuero y otros.

Realizar un programa de alimentación caprina en el nordeste argentino es difícil debido a que la vegetación natural, principal sustento de los rebaños, presenta una gran variación estacional en disponibilidad y en calidad, llegándose a extremos en algunos lugares donde los animales deben realizar grandes esfuerzos físicos para lograr sobrevivir a las penurias alimentarias, con la consecuente ineficiencia productiva y graves consecuencias socioeconómicas. Para ajustar la alimentación de un rebaño dentro de un sistema de producción determinado (extensivo, semi-intensivo o intensivo), es necesario realizar un diagnóstico sobre los aspectos nutricionales imperantes en cada lugar en particular y su metodología variará, obviamente, según el sistema de producción y el tipo de alimento disponible. 
La determinación e interpretación de ciertos indicadores bioquímicos es una valiosa herramienta diagnóstica que facilita evaluar el estado nutricional del rodeo permitiendo estimar en forma muy precisa el estado metabólico del animal. Grandes cambios del régimen pluviométrico inciden sobre la oferta forrajera de la pradera natural, ya que ocasiona una gran variabilidad estacional en cuanto a su calidad y cantidad. Este trabajo es parte de los resultados obtenidos en los dos primeros años de desarrollo de un proyecto referido al comportamiento estacional de algunos indicadores nutricionales en el ganado caprino de nuestra región.

\section{MATERIAL Y MÉTODOS}

El muestreo se efectuó en forma estacional sobre un total de 150 caprinos de distintas categorías, durante dos años consecutivos con registros pluviométricos muy diferentes: año 2008 con 950 mm y año 2009 con $1.490 \mathrm{~mm}$. Las muestras de sangre fueron obtenidas por venopunción yugular. Las determinaciones de analitos relacionados al metabolismo energético y nitrogenado fueron procesadas en el Laboratorio de Análisis Fisicoquímicos anexo a la Cátedra de Biofísica de la Facultad de Ciencias Veterinarias de la UNNE. Los estudios se efectuaron en espectrofotómetro de absorción molecular marca Perkin Elmer UV-Visible, modelo Lambda 25 y reactivos Wiener Lab.

Se realizaron las siguientes determinaciones: glucosa: método enzimático de glucosa-oxidasa/peroxidasa, lectura a $505 \mathrm{~nm}$; colesterol total: método enzimático específico de la oxidasa-peroxidasa, lectura a 505 $\mathrm{nm}$; colesterol ligado a lipoproteínas de alta densidad (HDL) y de baja densidad (LDL) por precipitación selectiva de la lipoproteína y valoración enzimática del colesterol; triglicéridos: método enzimático de la lipasa específica, lectura a $546 \mathrm{~nm}$; urea: método enzimático de la ureasa, lectura a $546 \mathrm{~nm}$; proteínas totales: método del Biuret, lectura a $540 \mathrm{~nm}$; albúminas: técnica de la bromocresol-sulfonftaleína, lectura a $625 \mathrm{~nm}$.

Los valores obtenidos se tabularon categóricamente en planillas de cálculos (Excel) y se procesaron estadísticamente mediante el software Infostat. De cada variable dependiente se verificó su normalidad distributiva a través del test de Wilk-Shapiro, lo que permitió el uso de estadísticas paramétricas de tendencia central (media aritmética) y dispersión (desvío estándar). La homogeneidad de la variancia se indagó por el test de Bartlett y el análisis de la variancia se realizó a una vía. Para todas las inferencias se estipuló $\alpha=5 \%$, por debajo del cual se rechazó la hipótesis nula de igualdad entre grupos.

\section{RESULTADOS Y DISCUSIÓN}

Las determinaciones químicas realizadas en forma estacional durante los dos años arrojaron los resultados que figuran en la Tabla 1. Los valores obtenidos según las diferentes categorías de animales no mostraron di- ferencias significativas entre sí, por lo que fueron evaluadas en forma conjunta según estaciones de un año respecto del otro.

Todos los parámetros del perfil lipídico se incrementaron durante el verano del año más lluvioso; colesterol HDL y colesterol total también lo hicieron en la primavera más seca y este último en el invierno más lluvioso. Colesterol LDL se incrementó en invierno del primer año y en otoño del segundo. En invierno y primavera del año más seco los animales acusaron descensos de proteínas totales y albúminas, respectivamente. La hipoglucemia se manifestó en el otoño e invierno más secos y durante el verano de ambos años.

El comportamiento estacional observado en algunos parámetros del metabolismo energético-proteico según las variaciones pluviales fueron importantes y deberían ser considerados en la evaluación nutricional del rodeo bajo situaciones similares en nuestra región. Gestación, parto y lactancia son considerados estados en los que el organismo está bajo un cierto grado de estrés metabólico y asociado a ello se han comunicado algunos cambios bioquímicos y hematológicos en distintas especies y razas de animales como es el caso de las cabras ${ }^{2}$, especialmente si cursan con un grado de coccidiosis importante ${ }^{11}$.

Las causas de abortos en cabras bajo pastoreo, si bien no se han estudiado en detalle, existe un consenso entre los investigadores que la mayor parte de ellos en el ganado caprino en sistemas extensivos tienen una etiología nutricional. Tanto en cabras de raza Angora ${ }^{30}$ como en cabras lecheras ${ }^{15}$ y mestizas ${ }^{22}$, se ha documentado que los animales que abortan por causas no infecciosas, presentan niveles sanguíneos de glucosa más bajos de lo normal y en estas condiciones se desencadena el proceso de expulsión del feto. En estas mismas razas ha comunicado un marcado incremento de abortos en animales con niveles deficitarios de energía ${ }^{16,29}$.

El metabolismo de las lipoproteínas es un proceso complejo, donde unas derivan de precursores aportados por otras lipoproteínas. En el bovino el proceso de formación de las lipoproteínas es similar al establecido en seres humanos, con algunas variantes ${ }^{3}$. Se ha descrito que la proteína que transfiere ésteres de colesterol y la lipasa hepática dependen del estado funcional de la glándula tiroides, incrementando su actividad en los casos de elevaciones subclínicas de la concentración hormonal tiroidea, provocando una reducción en la concentración de $\mathrm{HDL}^{9}$.

El contenido de selenio en la dieta se relaciona a las concentraciones de hormonas tiroideas aunque también pueden verse afectadas por factores diferentes, como son el consumo de yodo, grasas y al estado fisiológico del rumiante, entre otros ${ }^{1,20}$. Por lo tanto, su concentración obedece al resultado de múltiples factores y no se debería solo al consumo de selenio, aunque estudios en roedores han descrito el hallazgo de cambios en el metabolismo lipídico asociados con la concentración sérica de Se, con elevaciones del colesterol y LDL en individuos con concentraciones bajas del mineral ${ }^{14}$. 
Tabla 1: Valores obtenidos para los parámetros del perfil energético-proteico en caprinos del nordeste argentino según estación anual.

\begin{tabular}{|c|c|c|c|c|c|c|c|c|}
\hline \multirow[b]{2}{*}{ analito } & \multicolumn{2}{|c|}{ otoño } & \multicolumn{2}{|c|}{ invierno } & \multicolumn{2}{|c|}{ primavera } & \multicolumn{2}{|c|}{ verano } \\
\hline & $2008 n=23$ & $2009 \mathrm{n}=17$ & $2008 \mathrm{n}=16$ & $2009 \mathrm{n}=18$ & $2008 n=15$ & $2009 n=18$ & $08 \mathrm{n}=22$ & $2009 \mathrm{n}=21$ \\
\hline $\begin{array}{l}\text { coleste } \\
(\mathrm{g} / \mathrm{L})\end{array}$ & $\begin{array}{c}0,71 \pm 0 \\
\mathrm{~A}\end{array}$ & $\begin{array}{c}0,76 \pm 0,16 \\
A\end{array}$ & $\begin{array}{c}0,96 \pm 0,22 \\
\mathrm{~A}\end{array}$ & $\begin{array}{c}1,14 \pm 0,16 \\
\uparrow \mathrm{B}\end{array}$ & $\begin{array}{c}1,05 \pm 0,31 \\
\uparrow \mathrm{B}\end{array}$ & $\begin{array}{c}1,07 \pm 0,25 \\
B\end{array}$ & $\begin{array}{c}0,70 \pm 0,21 \\
\mathrm{~A}\end{array}$ & $\begin{array}{c}1,09 \pm 0,20 \\
\uparrow \mathrm{B}\end{array}$ \\
\hline $\begin{array}{l}\text { colesterol HDL } \\
(\mathrm{g} / \mathrm{L})\end{array}$ & $\begin{array}{c}0,52 \pm 0 \\
\mathrm{~A}\end{array}$ & $\begin{array}{l}53 \pm 0,15 \\
\mathrm{~A}\end{array}$ & $\begin{array}{c}0,50 \pm 0,11 \\
\mathrm{~A}\end{array}$ & $\begin{array}{c}0,61 \pm 0,07 \\
\mathrm{~A}\end{array}$ & $\begin{array}{c}0,79 \pm 0,13 \\
\uparrow \mathrm{B}\end{array}$ & $\begin{array}{c}0,65 \pm 0,11 \\
\mathrm{~A}\end{array}$ & $\begin{array}{c}0,53 \pm 0,14 \\
\mathrm{~A}\end{array}$ & $\begin{array}{c}0,76 \pm 0,13 \\
\uparrow \mathrm{B}\end{array}$ \\
\hline $\begin{array}{l}\text { colesterol L } \\
(\mathrm{g} / \mathrm{L})\end{array}$ & $\begin{array}{c}0,13 \pm 0,03 \\
\mathrm{~A}\end{array}$ & $\begin{array}{c}0,23 \pm 0,06 \\
\uparrow \mathrm{B}\end{array}$ & $\begin{array}{c}0,33 \pm 0,11 \\
\uparrow \mathrm{B}\end{array}$ & $\begin{array}{c}0,20 \pm 0,05 \\
\mathrm{~A}\end{array}$ & $\begin{array}{c}0,17 \pm 0,10 \\
\mathrm{~A}\end{array}$ & $\begin{array}{c}0,18 \pm 0,05 \\
\mathrm{~A}\end{array}$ & $\begin{array}{c}0,16 \pm 0,05 \\
\mathrm{~A}\end{array}$ & $\begin{array}{c}0,29 \pm 0,05 \\
\uparrow \mathrm{B}\end{array}$ \\
\hline $\begin{array}{l}\text { triglice } \\
(\mathrm{g} / \mathrm{L})\end{array}$ & $\begin{array}{c}0,21 \pm 0,06 \\
\mathrm{~A}\end{array}$ & $\begin{array}{c}0,31 \pm 0,13 \\
\mathrm{~A}\end{array}$ & $\begin{array}{c}0,30 \pm 0,13 \\
\mathrm{~A}\end{array}$ & $\begin{array}{c}0,32 \pm 0,11 \\
\mathrm{~A}\end{array}$ & $\begin{array}{c}0,35 \pm 0,10 \\
\mathrm{~A}\end{array}$ & $\begin{array}{c}0,31 \pm 0,11 \\
\mathrm{~A}\end{array}$ & $\begin{array}{c}0,56 \pm 0,23 \\
\uparrow \mathrm{B}\end{array}$ & $\begin{array}{c}0,50 \pm 0,12 \\
\uparrow \mathrm{B}\end{array}$ \\
\hline $\begin{array}{l}\text { proteínas totales } \\
(\mathrm{g} / \mathrm{dL})\end{array}$ & $\begin{array}{c}6,97 \pm 0,62 \\
\text { A }\end{array}$ & $\begin{array}{c}6,98 \pm 0,88 \\
A\end{array}$ & $\begin{array}{c}6,12 \pm 0,85 \\
\downarrow B\end{array}$ & $\begin{array}{c}7,50 \pm 0,89 \\
\mathrm{~A}\end{array}$ & $\begin{array}{c}7,12 \pm 0,72 \\
\mathrm{~A}\end{array}$ & $\begin{array}{c}7,11 \pm 0,55 \\
\mathrm{~A}\end{array}$ & $\begin{array}{c}7,74 \pm 0,90 \\
\mathrm{~A}\end{array}$ & $\begin{array}{c}7,47 \pm 0,82 \\
\mathrm{~A}\end{array}$ \\
\hline $\begin{array}{l}\text { albúminas } \\
\mathrm{g} / \mathrm{dL})\end{array}$ & $\begin{array}{c}3,60 \pm 0,42 \\
\mathrm{~A}\end{array}$ & $\begin{array}{c}3,53 \pm 0,56 \\
\mathrm{~A}\end{array}$ & $\begin{array}{c}3,18 \pm 0,36 \\
\mathrm{~A}\end{array}$ & $\begin{array}{c}3,01 \pm 0,42 \\
\mathrm{~A}\end{array}$ & $\begin{array}{c}2,84 \pm 0,23 \\
\downarrow \mathrm{B}\end{array}$ & $\begin{array}{c}3,59 \pm 0,48 \\
\mathrm{~A}\end{array}$ & $\begin{array}{c}3,63 \pm 0,52 \\
\mathrm{~A}\end{array}$ & $\begin{array}{c}2,99 \pm 0,33 \\
\mathrm{~A}\end{array}$ \\
\hline $\begin{array}{l}\text { rea } \\
g / L)\end{array}$ & $\begin{array}{c}0,28 \pm 0,08 \\
\mathrm{~A}\end{array}$ & $\begin{array}{c}0,31 \pm 0,05 \\
\mathrm{~A}\end{array}$ & $\begin{array}{c}0,34 \pm 0,08 \\
\mathrm{~A}\end{array}$ & $\begin{array}{c}0,31 \pm 0,05 \\
\mathrm{~A}\end{array}$ & $\begin{array}{c}0,29 \pm 0,08 \\
\text { A }\end{array}$ & $\begin{array}{c}0,26 \pm 0,06 \\
A\end{array}$ & $\begin{array}{c}0,31 \pm 0,06 \\
\mathrm{~A}\end{array}$ & $\begin{array}{c}0,23 \pm 0,06 \\
\mathrm{~A}\end{array}$ \\
\hline $\begin{array}{l}\text { glucosa } \\
(\mathrm{g} / \mathrm{L})\end{array}$ & $\begin{array}{c}0,37 \pm 0,08 \\
\downarrow \mathrm{B}\end{array}$ & $\begin{array}{c}0,45 \pm 0,11 \\
\mathrm{~A}\end{array}$ & $\begin{array}{c}0,30 \pm 0,09 \\
\downarrow \mathrm{B}\end{array}$ & $\begin{array}{c}0,45 \pm 0,11 \\
\mathrm{~A}\end{array}$ & $\begin{array}{c}0,47 \pm 0,12 \\
\mathrm{~A}\end{array}$ & $\begin{array}{c}0,45 \pm 0,11 \\
\mathrm{~A}\end{array}$ & $\begin{array}{c}0,39 \pm 0,08 \\
\downarrow \mathrm{B}\end{array}$ & $\begin{array}{c}0,34 \pm 0,08 \\
\downarrow \mathrm{B}\end{array}$ \\
\hline
\end{tabular}

Valores expresado en media artimética \pm desvío estándar. Letras distintas indican diferencias significativas $(\mathrm{p}<0.05)$.

Una dieta baja en Se no indujo cambios en la funcion tiroidea ni en el metabolismo de las lipoproteínas, excepto una mayor concentración de colesterol HDL ${ }^{21}$. Las hiperlipemias en bovinos pueden ser provocadas por aumentos de glucocorticoides y cursan con elevaciones en la concentraciones de colesterol y triglicéridos plasmáticos ${ }^{8}$. El comportamiento de las lipoproteínas es de gran valor diagnóstico ya que puede indicar alteraciones del metabolismo lipídico, por lo que deberían valorarse conjuntamente con el colesterol y los triglicéridos séricos.

Las variaciones en el metabolismo de las lipoproteínas también están relacionadas con factores diferentes a la función tiroidea; así, se han descrito cambios asociados con el estado nutricional y productivo del rumiante ${ }^{3}$, balance energético ${ }^{7,13}$, consumo de grasas en la dieta y cambios asociados en la respuesta a estímulos hormonales mientras dure el consumo de grasas ${ }^{7}$.

La síntesis de albúminas en el bovino se incrementaría por acción del cortisol aunque paralelamente aumentaría su tasa de degradación ${ }^{17}$. En animales se constataron disproteinemias, con catabolismo proteico incrementado debido a que la gluconeogénesis se realiza a expensas de los aminoácidos ${ }^{28}$. El nitrógeno no proteico, especialmente la urea, aumentaría en el hiperadrenocortisismo, aunque a veces permanecería enmascarado por la retención hídrica ${ }^{10}$.

Los valores aquí obtenidos constituyen un valioso auxiliar para conocer el estado nutricional de los rodeos caprinos del nordeste argentino. Mediante tales analitos se está evaluando el aprovechamiento real del alimento por parte del animal, ya que se lo estima en la última etapa de su asimilación, luego de haber sufrido diversas modificaciones en su digestibilidad, a través de efectos asociativos, competencia en su absorción, velocidad de tránsito intestinal, y otros factores. El per- fil metabólico puede ser aplicado al diagnóstico solo mediante el análisis estadístico con determinación de valores fisiológicos regionales de referencia, un buen conocimiento del alimento y agua de bebida, una adecuada capacidad interpretativa del dato bioquímico obtenido, muestreos frecuentes seriados y perfecto conocimiento del metabolismo y de sus interacciones ${ }^{4,5}$.

Se concluye que la variación estacional registrada en algunos indicadores del perfil energético-proteico estudiados podría deberse a cambios en la calidad y cantidad de la pastura ocasionados por las grandes diferencias pluviométricas registradas entre sendos años de ensayo.

\section{REFERENCIAS}

1. Arthur JR, Beckett GJ, Mitchell JH. 1999. The interactions between selenium and iodine deficiencies in man and animals. Nutr Res Rev 12: 55-73.

2. Azab ME, Hussein A, Abdel-Maksoud A. 1999. Changes in some hematological and biochemical parameters during prepartum and postpartum periods in female Baladi goats. Small Rum Res 34: 77-85.

3. Bauchart D. 1993. Lipid absorption and transport in ruminants. J Dairy Sci 76: 3864-3881.

4. Brem JJ. 1986. Perfiles bioquímicos en bovinos del centro-este de la Provincia de Formosa en distintos estadios productivos. Anales $12^{\circ}$ Congreso Argentino de Producción Animal, San Martín de los Andes (Argentina), Com. SA10.

5. Coppo JA, Pérez OA, Sandoval GL, Scorza SH. 1984. Perfiles bioquímicos en bovinos de Corrientes y provincias aledañas. Rev Milit Vet 32: 459-483.

6. De Ridder N, Benjamin RW, Van Keulen H. 1986. Forage selection and performance of sheep grazing dry annual range. J Arid Environ 10: 39-51. 
7. Drackley JK. 1999. Biology of dairy cows during the transition period: the final frontier? J Dairy Sci 82: 22592273.

8. Duncan JR, Prasse KW. 1986. Veterinary Laboratory Medicine. Clinical Pathology, 2nd ed., Iowa Univ. Press, Ames, 243 p.

9. Duntas LH. 2002. Thyroid disease and lipids. Thyroid 12: 287-293.

10. Espejo Rosas JL. 1988. Hallazgos clínicos de laboratorio, anatomopatológicos e histopatológicos en el hiperadrenocorticismo experimental. Vet Méx 19: 373-378.

11. Forchetti OD, Cuffre G, Amuchastegui JJ, Godio L. 1998. Indicadores sanguíneos del estado nutricional de las cabras. Prod Anim 18: 328-329.

12. Gall C. 1981. Goat production, Academic Press, New York, $619 \mathrm{p}$.

13. Grummer RR. 1993. Etiology of lipid-related metabolic disorders in periparturient dairy cows. J Dairy Sci 76: 3882-3896.

14. Huang K, Liu H, Chen Z, Xu H. 2002. Role of selenium in cytoprotection against cholesterol oxide-induced vascular damage in rats. Atherosclerosis 162: 137-144.

15. Hussain Q, Havrevoll O, Eik LO, Ropstad E. 1996. Effects of energy intake on plasma glucose, non-esterified fatty acids and acetoacetate concentration in pregnant goats. Small Rum Res 21: 89-96.

16. Hussain Q, Waldeland H, Havrevoll O, Eik LO, Andresen O, Engeland IV. 1996. Effect of type of roughage and energy level on reproductive performance of pregnant goats. Small Rum Res 21: 97-103.

17. Kaneko JJ. 1989. Clinical biochemistry of domestic animals, 4th ed., Academic Press, San Diego, 832 p.

18. Kenney PA, Black JL. 1984. Factors affecting diet selection by sheep. I Potential intake rate and acceptability of feed. Austr J Agric Res 35: 551-563.

19. Kouakou B, Gelaye S, Terrill T, Bennett J, Miller S, Amoah EA, Murry AC. 1997. Intake and digestion of le- guminous forages by mature goats, Publ. Am Forage and Grassland Council, Fort Valley (USA), p 207-211.

20. Matamoros R, Contreras PA, Wittwer F, Mayorga MI. 2003. Hipotiroidismo en rumiantes. Arch Med Vet 35: 1-11.

21. Matamoros R, Ceballos A, Contreras PA, Quiroga C, Andaur M, Wittwer F. 2008. Serum concentrations of triiodothyronine, thyroxine and lipid profile in dairy cows fed with a low selenium diet. Arch Med Vet 40: 23-29.

22. Mellado M, Valdez R, Lara LM, García JE. 2004. Risk factors affecting conception, abortion and kidding rates of goats under extensive conditions. Small Rum Res 55: 191198.

23. Morand-Fehr P, Sauvant D. 1984. Alimentación de cabras. En: Alimentos y alimentación de ganado (Church DC ed.), Hemisferio Sur, Montevideo, p. 553-577.

24. Papachristou TG, Papanastasis VP. 1994. Forage value of mediterranean deciduous woody fodder species and its implication to management of silvo-pastoral systems for goats. Agroforestry Syst 27: 269-282.

25. Papachristou TG, Nastis AS. 1996. Influence of deciduous broad-leaved woody species in goat nutrition during the dry season in northern Greece. Small Rum Res 20: 15-22.

26. Ramírez RG. 1989. Estudios nutricionales de las cabras en el noreste de México, Publ. Univ Autón Nueva León, México, $56 \mathrm{p}$.

27. Roig C. 2003. Alimentación del ganado caprino, Publ. Progano, INTA Colonia Benítez, Chaco (Argentina), 20 p.

28. Sodeman WA, Sodeman W. 1978. Fisiopatología clínica, $5^{\circ}$ ed., Interamericana, México, $952 \mathrm{p}$.

29. Van Der Westhuysen JM, Roelofse CS. 1971. Effect of shelter and different levels of dietary energy and protein on reproductive performance in Angora goats with special reference to the habitual aborter. Agroanimalia 3: 129-132.

30. Wentzel D, Le Roux MM, Botha LJ. 1976. Effect of the level of nutrition on blood glucose concentration and reproductive performance of pregnant Angora goats. Agroanimalia 8: 59-62. 